\title{
An Exploration of CUDA and CBEA for a Gravitational Wave Data- Analysis Application (Einstein@Home)
}

\author{
Jens Breitbart ${ }^{1} *$ and Gaurav Khanna ${ }^{2}$ \\ ${ }^{I}$ Research Group Programming Languages / Methodologies Universität Kassel Kassel, Germany \\ ${ }^{2}$ Physics Department, University of Massachusetts at Dartmouth North Dartmouth, MA, USA
}

\begin{abstract}
We present a detailed approach for making use of two new computer hardware architectures -- CBEA and CUDA -- for accelerating a scientific data-analysis application (Einstein@Home). Our results suggest that both the architectures suit the application quite well and the achievable performance in the same software developmental time-frame, is nearly identical.
\end{abstract}

Keywords: Astrophysics, gravitational wave, high performance computing, accelerators, CUDA, Cell Broadband Engine.

\section{INTRODUCTION}

The recent decades have brought a tremendous rise in computer simulations, in nearly every area of science and engineering. This is partly due to the development of (Beowulf) cluster computing that involves putting together "off-the-shelf" computing units (for example, commodity desktop computers) into a configuration that would achieve the same level of performance, or even outperform, traditional supercomputers at a fraction of the cost. Computational science has benefited and expanded tremendously in the last decade due to rapid improvements in CPU performance (Moore's Law) and major price drops due to mass production and intense competition.

However, a few years ago the computer industry hit a serious frequency wall, implying that increasing the processor's clock-rate for gains in performance could not be done indefinitely, due to rapid increases in power consumption and heat generation ( power wall). This led all the major processor manufacturers (such as Intel and AMD) toward multi-core processor designs. Today, nearly all commodity desktop and laptop processors are multi-core processors i. e. they combine two or more independent computing cores on a single chip. Thus, manufacturers today continue to pack more power in a processor, even though their clock-frequencies have not risen (and have stabilized at around $3 \mathrm{GHz}$ ). It is expected that this approach would work well for the next few years, but both Intel and AMD anticipate that even this approach will not scale well beyond 8 or 16 cores.

On the other hand, the overall performance of other computing technologies (e.g. graphics cards, gaming consoles, etc.) has continued to increase at a rapid rate, thus

*Address correspondence to this author at the Research Group Programming Languages / Methodologies Universität Kassel Kassel, Germany; Tel: +49-561-804-6268; Fax: +49-561-804-6219;

E-mail: jbreitbart@uni-kassel.de making general-purpose computing on such devices a tantalizing possibility. Both Compute Unified Device Architecture (CUDA) and Cell Broadband Engine Architecture (CBEA) are new hardware architectures designed to provide high performance and scaling for multiple hardware generations. CUDA is NVIDIA's generalpurpose software development system for graphics processing units (GPUs) and offers the programmability of their GPUs in the ubiquitous $\mathrm{C}$ programming language in conjunction with a set of libraries for memory management. The Cell Broadband Engine (CBE), which is the first incarnation of the CBEA, was designed by a collaboration between Sony, Toshiba, and IBM (so-called STI). The CBE was originally intended to be used in gaming consoles (namely Sony's Playstation 3) and consumer electronics devices, but the CBEA itself was not solely designed for this purpose and has been used in areas such as high-performance computing as well.

Both CUDA and CBEA are not the only new architecture approaches to overcome a decline in overall performance gains, but these are expected to be the ones with the widest distribution. CUDA is available for all NVIDIA GPUs based up on the G80 architecture and its successors, therefore all currently sold NVIDIA GPUs support CUDA. Sony has sold over 20 million Playstation 3 game consoles, each of which has a CBE main processor. Moreover, currently second to the fastest and the original peta-scale supercomputer is the IBM Roadrunner at LANL, which is a hybrid system, built using nearly 13,000 Cell processors and 6,500 AMD Opteron processors. Based on these successes, an industry standard -- OpenCL -- has been proposed by Apple and others, that would allow for computing across a variety of different hardware: CPU, GPU, CBE, etc. We consider the current distribution of this hardware an important factor for the future success of its underlying concept and programming model, and therefore we decided to work with these two architectures. 
In this article, we will compare the current state of both CUDA and the CBEA by modifying the Einstein@Home client application, to enable it to take advantage of these different hardware architectures. Einstein@Home [1] is a distributed computing project, that uses the computing power volunteered by end users running its client application, to perform data-analysis tasks for various gravitational-wave observatories such as LIGO and GEO. The computation performed by this client application can be executed in a data-parallel fashion, which suits both CUDA and the CBEA very well.

This article is organized as follows. First, Section 2 gives an overview of the Einstein@Home project and its client application. The next two sections, introduce the architecture and the software development system of the Cell Broadband Engine (Section 4) and our experiences with the development of the Einstein@Home client using it (Section 5). The following two sections describe CUDA (Section 6) and our experiences with that architecture (Section 7). In Section 8 we compare both implementations and outline the benefits and drawbacks of CUDA and the CBE and their respective software development systems. Section 9 discusses related work, while Section 10 provides a summary of our work.

\section{EINSTEIN@HOME}

Gravitational wave observatories are currently being built all over the world: LIGO in the United States, GEO/Virgo in Europe and TAMA in Japan. These will open a new window onto the Universe by enabling scientists to make astronomical observations using a new and significantly better medium -- gravitational waves (GWs), in addition to electromagnetic waves (light). These waves were predicted by Einstein's theory of General Relativity, but have not been directly observed because the required technology was simply not advanced enough, until very recently. Indirect evidence validating their existence has been available for some time i. e. the observed decay rate of the compact neutron star binary system PSR1913+16 [2] matches the theoretical predictions based on GW emission (a Nobel prize winning discovery!).

Using GWs as a medium for astronomical observation, alongside traditional approaches (light, neutrinos, etc.) holds tremendous promise not only in advancing our understanding of gravitational physics, compact astrophysical objects (like black holes and neutron stars) but also the entire Universe as a whole. The main reason why GWs are an excellent medium for doing astronomy, is that these waves interact extremely weakly with matter. This means that these waves can travel long distances, and even pass through large quantities of dust, gas, etc. without being significantly affected. Thus, they are able to transport valuable information over very long distances with little loss -- the key requirement for good astronomical observation. However, this very reason that makes them so good as an observational medium, also makes them notoriously hard to generate and detect. To produce a strong gravitational wave, one needs to move massive objects at very high accelerations -- collisions between compact astrophysical objects are therefore, the ideal candidates. But even for the scenario of two solar-mass black holes colliding at a distance of (say)
$100 \mathrm{Mpc}$ away, the "strain" caused by the emitted GW that we would need to measure on Earth for a successful detection is of the order of $10^{-21}$. In other words, one would need to be able to detect fluctuations in length that are less than the size of a nucleus of an atom, over the scale of kilometers! This is precisely why extremely sophisticated technologies (see [3] for a recent review) have to be developed to be able to make such a detection a reality. Over the next few years, GWs will be detected and a wealth of new astronomical data will begin pouring in $[4,5]$. That will allow us to not only learn more about black holes and neutron stars, but also test our theories (for example, General Relativity) in extremely strong-field regimes where they have never been tested before. Moreover, this would even help to rule out or establish, other alternative theories of gravitation [6]. For all these reasons, the scientific community worldwide, has invested a tremendous amount of time, energy and resources in developing the observatories, theory, and data analysis techniques necessary to make GW astronomy a possibly in this decade.

These observatories generate data at the rate of several tens of GBs per day and they require highly computationally intensive data-analysis (mainly due to the fact that the signal-to-noise ratio in the data streams is very low). Einstein@Home is a BOINC [7] based, public distributed computing project that offloads the data-analysis associated to these observatories to volunteers worldwide. The project currently (since 2005) has over 200,000 participants and over 800,000 computers involved, with a strong and sustained growth pattern. The goal of Einstein@Home is finding gravitational waves emitted from neutron stars ( pulsars), by running a brute force search for different waveforms in an extremely large data-set.

We consider the Einstein@Home client a meaningful test application for our comparison of CUDA and the CBE since from a theoretical viewpoint, its parallelization is quite straightforward. Furthermore, the application is very compute intensive, therefore we can expect a high performance gain through parallelization. The computation of the application can be roughly divided into two parts -the so-called F-Statistics computation, and a Houghtransformation. We will only concentrate on the F-Statistics computation in this work, and provide below a brief overview of the algorithm and its data dependencies; a detailed discussion can be found in [8].

Listing 2 provides an overview of the F-Statistics code. The code uses for each whenever the loop can be carried out in parallel with no or minimal changes to the loop body. + is used for all commutative calculations. The parameters of a function denote all the values a function depends on, however we use "..." as a reference to all parameters passed to the called function. The F-Statistics code consist of multiple nested loops, each looping through a different part of the input data set, namely: a frequency band, all used detectors for the observing the gravitational wave and an SFT of each signal.

T ComputeFStatFreqBand(...) \{

T result[];

int $\mathrm{i}=0$; 
for each (frequency in frequency_band(...))

result $[i++]=$ ComputeFStat $(\ldots$, frequency $)$;

return result;

\}

T ComputeFStat (...) \{

$\mathrm{T}$ result;

for each (detector in detectors)

result $+=$ ComputeFaFb(..., detector $)$;

return result;

\}

$\mathrm{T}$ ComputeFaFb(...) \{

$\mathrm{T}$ result;

for each (SFT in SFTs(frequency))

result $+=$ some_calculations $(. . .$, SFT $)$;

return normalized(result);

\}

In this article we will indicate the current state of the calculation, as the presently computed upon SFT, detector and frequency -- or more formally speaking: the current state is the tuple of (frequency, detector, signal) using the variable names used in the Listing 2.

For simplicity, Listing 2 does not include any data structures for storing data. Here we provide a very brief introduction (a more detailed overview can again be found in [8]). The data structures used by Einstein@Home are based on a deep hierarchy. For example, the data structure that is used to store the SFT data for one frequency, is called MultiSFT. MultiSFT has a pointer to a data array, which stores pointers to the SFT data for every detector. The SFT data is built of two levels, thus to finally access the data one needs code similar to the one shown in Listing 2. The additional two layers within the SFT data are a consequence of how the data is stored and processed within the some_calculations function. We will not go into more detail here, since the rest consists of calculations that must be executed sequentially and are therefore not important for the parallelization.

[caption=Pseudo code showing the SFT data structure] MultiSFT x;

float data $=$ x. data $[$ detector $]->$ data $[$ SFT] $->$ data $[\mathrm{i}]->$ data $[\mathrm{j}]$;

\section{TEST CASES}

The various measurements presented in this article were done with two different kinds of data-sets that not only differ in the overall runtime, but also in their memory requirements and performance characteristics. The small test case is based on a data-set used to check if the Einstein@Home client application itself is producing correct results. It uses a small number of frequencies in contrast to a full Einstein@Home work unit. In the small data set case, the F-Statistics takes nearly $90 \%$ of the overall runtime, while in the full work unit case its share is nearly $50 \%$. A full work unit consists of multiple, so-called sky points. The calculation done for one sky point consists of both the F-Statistics and the Hough transformation and the runtime required to compute one sky point is nearly identical to the one required for the other sky points. For this work, we therefore do not measure the runtime of a full work unit, but only measure the time required to calculate one sky point.

\section{CELL BROADBAND ENGINE}

The CBE is a completely redesigned processor, that was developed collaboratively by Sony, IBM and Toshiba mainly for multimedia applications. This processor has a general purpose (PowerPC) CPU, called the PPE (that can run 2 software threads simultaneously) and 8 special-purpose compute engines, called SPEs available for raw numerical computation. Each SPE can perform vector operations, which implies that it can compute on multiple data, in a single instruction (SIMD). All these compute elements are connected to each other through a high-speed interconnect bus (EIB). Note that the design of this processor is very different from traditional multi-core processors. In a certain sense, the CBE's design is somewhere between a generalpurpose CPU and a specialized GPU (as described in Section 6). It can therefore be considered as a hybrid technology, having the advantages of both these architectures. The outcome of this distinctive design is that a single, $3.2 \mathrm{GHz}$ (original - 2006/2007) CBE has a peak performance of over 200 GFLOPS in single-precision floating point computation, and 15 GFLOPS in double-precision. It should be noted that the current (2008) release of the CBE, called the PowerXCell, has design improvements that bring the doubleprecision performance up to 100 GFLOPS.

We will not attempt to go into much more detail concerning the CBE's design here, rather we will simply point out one unique feature that addresses the issue of the memory wall that is common to all current computer hardware. The memory wall refers to the large (and increasing) gap between processor and memory performance, causing the slower memory speeds to become a significant bottleneck. The current state-of-the-art approach to combat this issue has been to include large cache sizes (several MBs) on the processor chip. However, this takes away valuable space (for compute elements) on the chip, and thus may result in only a marginal, overall performance increase. A key feature of the the $\mathrm{CBE}$ is its unique ability to interleave computation and data access. Therefore, it is possible for the programmer to overlap memory access and the actual computation ("double buffering"), in order to hide the time it takes to access memory. It is this mechanism that allows the $\mathrm{CBE}$ to break through the memory wall and perform very efficiently, even for computations that have a large memory footprint. It is also partly for this reason, that the CBE can reach a "real world" application performance that is nearly $100 \%$ of its theoretical peak performance [9]. The parallel programming model on CBEA allows for the use of SPEs for performing different tasks in a workflow ("task parallel" model) or performing the same task on different data ("data parallel" model). We use the data parallel model in our implementations.

One (software) challenge introduced by this new design, is that the programmer has to explicitly manage the memory transfer between the PPE and the SPEs. The PPE and SPEs 
are equipped with a DMA engine -- a mechanism that enables data transfer to and from main memory and each other. Now, the PPE can access main memory directly, but the SPEs can only directly access their own, rather limited (256KB) local store. This poses a challenge for some applications, including the Einstein@Home client application that we are considering in this article. However, compilers (IBM XLC/C++) are now available that enable a software caching mechanism that allow for the use of the SPE local store as a conventional cache, thus negating the need of transferring data manually from main memory. Another important mechanism that allows communication between the the different elements (PPE, SPEs) of the CBE is the use of mailboxes. These are special purpose registers that can be used for uni-directional communication. Each SPE has three mailboxes -- two outbound, that can hold only a single entry, and one inbound, that can hold four entries. These are typically used for synchronizing the computation across the SPEs and the PPE, and that is primarily how we made use of these registers as well. Details on our specific use of these various aspects of the CBE for the Einstein@Home client application appear next section of this article.

\section{IMPLEMENTATION ON THE CELL BROADBAND ENGINE}

As it can be seen in Listing 2 the F-Statistics calculation consist of multiple nested loops that can be carried out in parallel. All loop iterations in the function ComputeFStatFreqBand can be carried out with no changes, whereas both the loops in the functions ComputeFStat and ComputeFaFb execute a reduction. We parallelized the FStatistics code by parallelizing the loop in ComputeFStatFreqBand. We did this because parallelizing the outer loop limits the overhead generated by the parallelization and the number of frequencies is an order-ofmagnitude higher than the number of detectors or the SFTs. The parallelization is done by equally assigning a number of frequencies to the SPEs independently, and having each SPE calculate the results for the assigned frequencies. The PPE is only used for synchronizing the SPEs -- the PPE tells the SPEs when to start with the calculations and waits for all the SPEs to finish their calculations before continuing with the Einstein@Home application. The code executed by the SPEs is simply a copy-and-paste of the original code, except for the modification that we describe below.

We developed two F-Statistics implementations for the CBE. In the first implementation, we manually manage transfers to and from the local store of the SPEs, whereas the second implementation relies on the software cache implementation provided by IBMs XLC/C++ compiler. In this section, we will first describe the two implementations and discuss the benefits of each later. We will refer to the first implementation as DMA-Fstat, whereas the second implementation will be called EA-Fstat.

As we stated above, DMA-Fstat uses manual DMA data transfers, therefore the first step of the development for this implementation was to make sure that the data that must be transferred to the local store, complies with the memory alignment requirements for the DMA transfers of the CBE. The IBM Cell SDK includes special malloc-like functions, that return memory which is properly aligned for such DMA transfers. Imposing the memory alignment requirements for all data structures was done by modifying Einstein@Home client malloc function wrappers, so they call the special malloc functions provided by the SDK. Measurements show that using the SDK's malloc function for all memory allocations, does not cause any performance issues, therefore we decided to allocate all data structures with DMAcomplying memory alignments, even though it is required only for data structures that need to be transferred to the SPE local store. Furthermore, identifying the part of the application, where the data structures used by the SPEs are allocated would require a more detailed knowledge of the Einstein@Home client than we currently have.

The DMA-Fstat build of the client is based on the well known thread programming paradigm. The PPE creates multiple threads, each of which is used to control a single SPE. After the threads are created, the PPE inputs the data structure addresses used by F-Statistics into the mailboxes of the SPEs. This communication is also used to notify the SPEs to begin work. After the SPEs have received the addresses, they use DMA transfers to get all data required for the complete computation. We cannot use double buffering because the data that is needed for the calculation is computed on the fly for most data structures. We could have implemented double buffering for some data structures, but we did not do so, because DMA-Fstat cannot be used for a full work unit anyway (explained below). Since we did not use double buffering, that diminishes the possible performance gain we could achieve with this implementation. Moreover, the need to transfer all data at the beginning of the calculation, in conjunction with the rather small size of the local store available on the SPEs limits the amount of data that can be processed by each SPE. DMAFstat works well for the small data set case, but is unable to process the full data set. After they complete the computation, the SPEs write their results back to main memory by using DMA transfers and place a "work finished" message in the mailbox. The PPE waits until all SPEs have placed this message in their mailbox, before the Einstein@Home client is executed any further.

We developed our second F-Statistics implementation (EA-Fstat) to no longer be limited by the amount of data that can be processed. EA-Fstat relies on the SPE software cache implementation of the $\mathrm{XLC} / \mathrm{C}++$ compiler, that freed us from manually transferring data to the local store. Furthermore, we did not need to guarantee any memory alignment for the data that must be accessed by the SPE. We only needed to guarantee that in the SPE code all pointers, pointing to main memory are qualified with the _-ea qualifier. Since the data structures used by the Einstein@Home client are deeply pointer based, this modification took some time, but was far less intrusive than changing the memory alignment of all data structures, as was done in DMA-Fstat. The initial communication is done identically as in DMA-Fstat, meaning that the addresses of the data structures in main memory are sent to the SPE mailboxes. These addresses are assigned to _ea qualified pointers and then used as if they point to locations in the SPE's local store. The synchronization of SPE and PPE is again done identically to that of DMA-Fstat, however before the SPE sends out the "work finished" message in the 
Table 1. The Time Required for the CBE Client Running the Small Test Case

\begin{tabular}{|c|c|c|}
\hline PPE & DMA-Fstat & EA-Fstat \\
\hline \hline $8: 53 \mathrm{~min}$ & $2: 30 \mathrm{~min}$ & $2: 34 \mathrm{~min}$ \\
\hline
\end{tabular}

mailbox, the SPE writes back the data stored in the local store cache. The cache write back is done by manually calling a special function.

The benefit of EA-Fstat compared to DMA-Fstat is that the developer no longer has to worry about the size of the local store and can automatically benefit from larger local store in future hardware generations. Furthermore, relying on the software cache reduces the complexity of the SPE program code: DMA-Fstat required 122 lines of code (not counting comments) consisting of memory allocation and pointer arithmetic, whereas the EA-Fstat implementation only consists of 9 library function calls, that read data structure addresses out of the mailboxes.

Table 1 shows the runtime of the original Einstein@Home client using only the PPE in comparision with both our new implementations, for the small work unit case. These measurements were performed by running the codes on a Sony Playstation 3, that allows us to use a maximum of 6 SPEs. Both DMA-Fstat and EA-Fstat use only 3 SPEs, since the small amount of work of the small test case does not scale well with additional SPEs. We can see that the PPE is clearly outperformed by the clients that use the SPEs. This should come at no surprise since those clients run the F-Statistics calculation in parallel on multiple SPEs without any need for synchronization. Furthermore, we can store all the data that is required for the F-Statistics computation in the fast local store of the SPE, thus there is no need to access slow main memory. The comparision of both DMA-Fstat and EA-Fstat shows the performance hit of using the software cache mechanism is only about $2.5 \%$ in our small test case. The low performance loss is partly due to the fact that we did not use double buffering, which would have likely increased the performance of DMA-Fstat.

We show the performance of our EA-Fstat solution in Table 2. When running a full work unit the EA-Fstat client cannot outperform the PPE version as well as it did in the small data-set test case. When running the client with 6 SPEs, the client finishes in about $59 \%$ of the runtime of the PPE-only client -- we gain a factor of 1.7 in overall application performance, by making use of the CBE architecture. The reduced performance gain does not seem to be a result of the size of the used software cache -- halving the cache size to $64 \mathrm{~KB}$ reduces the performance by $2 \%$. The performance of the EA-Fstat client is limited by the runtime required by the Hough transformation, which turns out to be the performance bottleneck when running the client with 6 SPEs. When considering the F-Statistics computation alone, the performance improved by a factor of about 5.5 upon using 6 SPEs -- F-Statistics requires less than two minutes of the overall runtime.

The best overall performance of the Einstein@Home client on the CBE platform is probably achieved by running multiple clients on one system, so the PPE's ability of running 2 software threads can be used and all SPEs are kept busy, even when one client is currently executing the Hough transformation. Our experimentation suggests that one can gain an additional 30\% in overall application performance in this manner. It may also be possible to parallelize the Hough computation, so that it utilizes the SPEs. Further work is required to identify, which solution is the best option.

\section{CUDA ARCHITECTURE}

CUDA is a general-purpose programming system only available for NVIDIA GPUs and was first publicly released in late 2007. CUDA requires a GPU that is based on NVIDIA's so-called G80 architecture or one of its successors. Through CUDA, the GPU (called device) is exposed to the CPU (called host) as a co-processor with its own memory. The device executes a function (called kernel) in the SPMD model, which means that a user-configured number of threads runs the same program on different data. Threads executing a kernel must be organized within so called thread blocks, which may consist of up to 512 threads; multiple thread blocks are organized in a grid, which may consist of up to $2^{17}$ thread blocks. Thread blocks, however, are not there purely for organizational purposes, they also play an important role related to performance. Each thread block is always scheduled onto one so-called multiprocessor of the device. A single multiprocessor consists of 8 processors. The number of multiprocessors of a device depends on hardware used with the current maximum of multiprocessors on a single device being 30 . If a kernel uses only one thread block, only one multiprocessor of the device is used and most of the devices' processing power is not utilized. Multiple thread blocks can be assigned to the same multiprocessor, in which case, they equally share the resources of the multiprocessor. Thread blocks are important for algorithm design, since only threads within a thread block can be synchronized. NVIDIA suggests having at least 64 threads in one thread block and up to multiple thousands of thread blocks -- more threads than the device has processors -- to achieve high performance. Threads within thread blocks can be addressed with one-, two- or threedimensional indexes; thread blocks within a grid can addressed with one- or two-dimensional indexes. We call the thread index threadIdx and the dimensions of the thread

Table 2. Time Per Sky Point for the CBE Client for a Full Work Unit Case

\begin{tabular}{|c|c|c|c|c|c|c|}
\hline \multirow[b]{2}{*}{ PPE } & \multicolumn{6}{|c|}{ Processing Elements } \\
\hline & \multicolumn{6}{|c|}{ SPEs } \\
\hline $22 \mathrm{~min}$ & $20 \mathrm{~min}$ & $16 \min$ & $14.5 \mathrm{~min}$ & $13.75 \mathrm{~min}$ & $13.5 \mathrm{~min}$ & $13 \mathrm{~min}$ \\
\hline
\end{tabular}


Table 3. Time Measurements of the CUDA Based Client

\begin{tabular}{|c|c|c|}
\hline Test Case & CPU & GPU \\
\hline \hline small & $2: 19 \mathrm{~min}$ & $1: 22 \mathrm{~min}$ \\
\hline full (per sky point) & $11: 00 \mathrm{~min}$ & $7: 00 \mathrm{~min}$ \\
\hline
\end{tabular}

block $\mathrm{x}, \mathrm{y}$ and $\mathrm{z}$ (therefore threadIdx. $\mathrm{x}$ is the first dimension of the thread index). We refer to the thread block index as blockIdx and use the same names for the dimensions.

From the host's point of view, kernel invocations are asynchronous function calls. Synchronisation between host and device are done explicitly by calling a synchronization function, or implicitly when the host tries to access memory on the device. In both cases, synchronization takes the form of a barrier that blocks the calling host thread until all previously called kernels are finished.

In contrast to main memory used by the CPU, its GPU counterpart -- called global memory -- is not cached and accessing it costs an order-of-magnitude more than most calculations. For example, 32 threads require 400 - 600 clock cycles for a read from global memory, whereas an addition executed by the same number of threads takes only 4 clock cycles. The high cost of accessing global memory is another incarnation of the so-called memory wall that also was an important factor for the design of the CBE (see Section 4). However, NVIDIA chose another way to circumvent this problem. The device uses an efficient thread scheduler that uses the massive parallelism approach of the device to hide the latency by removing threads that just issued a global memory read from its processor and scheduling a thread that is not waiting for data. This is one of the reasons why the device requires more threads than there are processors available to achieve good performance. Furthermore, CUDA provides a way of directly reducing global memory accesses, by using a special kind of memory called shared memory. Shared memory is fast memory located on the multiprocessors of the device itself and is shared by all threads of a thread block. Accessing shared memory costs about 4 clock cycles for 32 threads and may be used as a cache, which must be managed by the developer. However, using global memory cannot be avoided, because it is the only kind of memory, which can be accessed by both the host and the device. Data that is stored in main memory must be copied from main memory to global memory by a CUDA memcopy function call, if it is needed by the device. Results of a kernel that need to be used by the CPU must be stored in global memory and the CPU must issue a memcopy from global memory to main memory to use it. All transfers done by CUDA memcopy functions are DMA transfers and have a rather high cost of initialization and a rather low cost for transferring the data itself.

\section{IMPLEMENTATION USING CUDA}

The development of the CUDA based F-Statistics was an evolutionary process. We developed three different versions, each solving the problems that emerged in the previous version.

We decided to start our work by porting the innermost function of the F-Statistics (ComputeFaFb) to the device.
Porting the code to be executed by the device did not require any difficult work. The implementation uses one thread per SFT and only one thread block. The number of SFTs in our data sets are less than the maximum number of threads in one thread block. Since we use one thread per SFT, we calculate all the SFTs of one detector (and therefore, all SFTs processed by one call of ComputeFaFb) in parallel. More formally speaking, we calculate the states (frequency, detector, threadIdx.x) in one kernel call, with threadIdx.x being the $\mathrm{x}$-dimension of the block local CUDA thread index.

The reduction which is part of ComputeFaFb, is implemented by using synchronized access to shared memory, which is possible since all threads are in one thread block. Reductions, that include multiple thread blocks are difficult to achieve with this hardware, since one cannot synchronize threads of different thread blocks or support hardware floating point atomic operations. Therefore, we did not implement a parallel reduction. A parallel reduction example is provided by NVIDIA [10], so we can easily replace our implementation in the future. In our implementation, the first thread collects all the data from the other threads, calculates the result and writes it to global memory. The performance of this first version is not limited by the performance of the calculations done at the device, but by the device memory management done by the host. We refer to this implementation as version 1.

The concept behind version 1 was simply to start our work in a straightforward way, and not necessarily obtain better performance than the CPU version. Nonetheless, we expected the overall performance to be half that of the hostbased implementation, but version 1 takes more than 70 times the runtime of the CPU version! Measurements show that almost $95 \%$ of the runtime is spent on device memory management and about $70 \%$ on copying the data to and from the device. This problem arises because version 1 uses the same data structure as the original CPU version, which consists of a large number of small memory blocks (the exact number depends on the data). To transfer the data structure directly to the device, version 1 needs to issue an DMA transfer to global memory for every memory block. Since a DMA transfer has a rather high cost of initialization and a rather low cost for transferring a data element itself, this data structure design simply cannot provide high performance.

Our second implementation (version 2) still calculates the states (frequency, detector, threadIdx.x) in one function call, but uses a newly developed data structure. The new data structure was designed to directly benefit from the way DMA memory transfers work. In the kernel, the data is typically read once from a known position that only depends on the current state of the calculation. The requirements of both fast memory transfer and fast access to an element at an arbitrary but known position are satisfied by simply using an array. An array is guaranteed to be stored in one contiguous memory block and each element can be directly accessed by using an index, its address, or a combination of both i. e. an offset based on an address pointing to one element in the array. Thus, our new data structure is an aggregation of a small number of arrays. 
These arrays can be grouped in two types. One type stores data used to calculate the result of the F-Statistics and is therefore called data arrays. The other type stores pointers, that point to elements inside the data arrays -- we refer to this type as offset arrays, since the pointers stored inside these arrays are used as an offset to access data the way we have just described. The creation of this data structure itself is rather simple. The original small memory regions are all padded into a data array in an ordered fashion. The elements in the offset arrays are used to identify the original memory regions. The new data structure is only used by the device, the code executed at the host continues to be the previous one.

Performance measurements of version 2 show that our new data structure solves the problems of version 1. Memory management is still a measurable amount but is no longer the performance bottleneck. Version 2 has approximately the same performance as the original CPU version. Next, we decided to change our kernel call concept, as used by version 1 , to utilize multiple multiprocessors of the device and thus improve performance (we had repeatedly issued kernel calls with only one thread block so far). Below, we describe how we replaced these multiple calls with just one kernel call using multiple thread blocks.

Let us first revise the important details of Listing 2 before we explain how we implemented the final version of F-Statistics with CUDA. If we want to calculate the FStatistics for a frequency band, we have to port the functions ComputeFStatFreqBand and ComputeFStat to the device. ComputeFStat contains a reduction of the values calculates by ComputeFaFb -- the function we ported to the device in step one. The results of ComputeFStat are written to different memory addresses. As previously described, we use a fairly simple way to calculate the reduction done in ComputeFaFb, which is possible only because all threads are in the same thread block. Our analysis of the used data sets showed that we could continue with this approach. The number of threads required to calculate ComputeFaFb for all detectors is still small enough to have one thread block calculate them. For easy readability of our code we use two dimensional thread blocks. The $\mathrm{x}$-dimension still represents the calculated SFT, whereas the y-dimension is now used to represent the detectors. Version 1 calculates the states (frequency, detector, threadIdx.x) with one kernel call, whereas our re-worked approach calculates (frequency, threadIdx.y,threadIdx.x) with one kernel call. Even though this approach uses more threads, it still uses only one thread block per kernel call.

The next step is to use multiple thread blocks to calculate different frequencies i.e. to calculate the states (blockIdx.x,threadIdx.y, threadIdx.x) with one kernel call. Conceptually this can be achieved with relative ease, since the calculations of one frequency are independent from one another. Nonetheless, this concept still results in some overhead work that may not be very obvious at first. For example, the number of signals is not constant across all frequencies, but the dimension of all thread blocks is identical. Therefore, we first scan the data-set for the maximum number of signals found in a frequency of a given frequency band and thereby determine the x-dimension of all thread blocks. This approach results in idle threads for all the other thread blocks, but our evaluation of the dataset shows only a small fraction of idle threads -- typically, 6 threads idle per $\mathrm{x}$-dimension, with the maximum being 10 . This is our final implementation of the Einstein@Home CUDA application, which we will refer to as version 3 . The development of all three versions was done on a device, that did not support double-precision floating point operations which are required by Einstein@Home to produce correct results. This client therefore, does not use double-precision and the results are of no scientific value.

Upon running our implementation on a test system with a GeForce GTX 280 and two AMD Opteron 270 (2 GHz) the CUDA version performs about 1.7 times as fast as the CPU version for the small test case. When run on a full work unit, the CUDA client performs about 1.6 times as fast as the CPU version. Note that both the CPU and the GPU version only use one core when running the Einstein@Home client. Considering only F-Statistics, the performance was improved by more than a factor of about 3.5 -- the FStatistics calculation only takes about 1.5 minutes.

\section{COMPARISION OF CUDA AND THE CBE}

One of the most important factors when developing for the CBEA is the size of local store, especially when the developer needs to manually manage the data storage in the local store. The data required by our application does not fit in the local store. An easy way to overcome this is to use a software cache -- however, by using this technique, the developer loses the chance to utilize double buffering, which is one of the most important benefits of the CBEA. Using CUDA required us to change the underlying data structure of our application, which we could also use with the CBEA. In our case the data structure redesign was rather easy, however in other cases this may be more problematic. The main benefit of using CUDA is the hardware, that increases performance at a much higher rate than multi-core CPUs or the CBEA. Furthermore, software written for CUDA can easily scale with new hardware -- at least when a high number of thread blocks is used. However, writing software that achieves close-to-the-maximum performance with CUDA is very different when compared to most other programming systems, since thread synchronization or calculations are typically not the time consuming parts, but memory accesses to global memory are. We did not optimize any of our implementations to the maximum possible performance, instead invested a similar amount of development time into both the CBE and the CUDA client. Therefore, our performance measurements should not be considered as what is possible with the hardware, but rather what performance can be achieved within a reasonable development time-frame.

\section{RELATED WORK}

Scherl et al. [11] compare CUDA and the CBEA for the so called FDK method for which CUDA seems to be the better option. Christen et al. [12] explore the usage of CUDA and the CBEA for stencil-based computation, which however does not give a clear winner. In contrast, our work does not strive for the maximum possible performance and relies on very low cost hardware. 


\section{CONCLUSION / FUTURE WORK}

The main outcome of our work presented in this article, is that new upcoming architectures such as CBEA and CUDA have very strong potential for significant performance gains in scientific computing. In our work, we focussed on a specific, data-analysis application from the gravitational physics community, called Einstein@Home. This is amongst the largest BOINC-based, public distributed computing projects. Using these architectures, we successfully accelerated by several fold, one of the two computationally intensive routines of the Einstein@Home client application. Our final CBEA and CUDA implementations yield comparable performance, although the development process, software tools, challenges faced, etc. relevant to these two architectures were completely different. In this article, we described our experiences with this software development in great detail.

From the point of view of the Einstein@Home project, we expect our work to have a major impact. The CUDA based GPU client will ultimately enable the project to harness the vast computing power currently inaccessible in consumer desktop and laptop computer graphics cards. When the GPU client is deployed, we anticipate the total computing power of the project to increase substantially. The CBE client would similarly be extremely helpful to the project. There are currently 20 million Playstation 3 gaming consoles sporting the CBE that could potentially compute for Einstein@Home once our client application is deployed by the project. That would likely increase the overall computing power of Einstein@Home many fold. It is worth noting that the release of a similar Playstation 3 client enabled Stanford's Folding@Home to quantum leap into the petascale regime in 2007 [13].

In the future, we expect to complete the final development of the CBEA and GPU clients and address some of the issues they currently have (as described before in this article). We will also investigate the use of GPU and CBE optimized versions of the Hough computation to accelerate the clients even further (recall, that the Hough computation is now the most significant bottleneck for these clients). Lastly, we will also work closely with the Einstein@Home project team to integrate our CBEA and GPU clients alongside the currently operational CPU clients, for a full-scale deployment.

\section{ACKNOWLEDGMENT}

The authors would like to thank NVIDIA and Sony for providing the hardware that was used for development, testing and benchmarking our codes. Gaurav Khanna would also like to acknowledge support from the National Science Foundation (grant number: PHY-0831631).

\section{REFERENCES}

[1] Einstein@Home website. http://einstein.phys.uwm.edu

[2] Hulse RA, Taylor JH. Discovery of a pulsar in a binary system. Astrophys J Lett 1975; 195:151.

[3] Hello P, Whelan JT, Woan G, Fairhurst S, Guidi GM. Current status of gravitational-wave observations. Gen Rel Grav 2011; 43: 387.

[4] Smith JR, LIGO Scientific Collaboration. The path to the enhanced and advanced LIGO gravitational-wave detectors. Class Quant Gravity 2009; 26(11): 114013 .

[5] LIGO Scientific Collaboration. The 8th Amaldi Conference on Gravitational Waves (Amaldi 8); Class Quant Grav 2010.

[6] Corda C. Interferometric detection of gravitational waves: the definitive test for general relativity. Int J Mod Phys 2009; D18(14): 2275.

[7] Anderson DP. BOINC: A system for public-resource computing and storage. In: Proceedings of the 5th IEEE/ACM International Workshop on Grid Computing. Washington, DC, USA: IEEE Computer Society 2004.

[8] Breitbart J. Case studies on GPU usage and data structure design. Master's thesis. Germany: University of Kassel 2008.

[9] Hackenberg D. Fast Matrix Multiplication on Cell (SMP) Systems 2009. http://www.tu-dresden.de/zih/cell/matmul

[10] NVIDIA Corporation. CUDA parallel reduction [URL]; 2007; http://developer.download.nvidia.com/compute/cuda/1_1/Website/ Data-Parallel_Algorithms.html

[11] Scherl H, Keck B, Kowarschik M, Hornegger J. Fast gpu-based ct reconstruction using the Common Unified Device Architecture (CUDA). In: Eric C. Frey, Ed. USA: Nuclear Science Symposium, Medical Imaging Conference 2007.

[12] Christen M, Schenk O, Messmer P, Neufeld E, Burkhart H. Accelerating stencil-based computations by increased temporal locality on modern multi- and many-core architectures. In: New Frontiers in High-performance and Hardware-aware Computing (HipHaC). Italy 2008.

[13] Folding@Home website. http://folding.stanford.edu 